\title{
Os Modelos Internos Dinâmicos Analisados com Recurso às Narrativas de Representação da Vinculação em Adultos
}

\author{
Ligia Monteiro \\ Instituto Universitário de Lisboa (ISCTE-IUL), CIS-IUL, Lisboa Portugal \\ Manuela Veríssimo ${ }^{1}$ \\ Filipa Silva
}

ISPA - Instituto Universitário de Ciências Psicológicas, Sociais e da Vida, Lisboa, Portugal

Irina Branco

Instituto Universitário de Lisboa (ISCTE-IUL), Lisboa Portugal

António J. Santos

ISPA - Instituto Universitário de Ciências Psicológicas, Sociais e da Vida, Lisboa, Portugal

\section{Resumo}

Este estudo analisou os modelos internos dinâmicos de mães recorrendo às Narrativas de Representação da Vinculação em Adultos (Waters \& Rodrigues-Doolabh, 2004). Teve como objetivos: testar a validade interna e discriminativa do instrumento analisando, ainda, os aspectos estilísticos das narrativas produzidas por 89 mães com crianças a frequentar Creche/Jardim-de-Infância. As narrativas foram recolhidas em sessões individuais tendo sido posteriormente transcritas e cotadas por dois a três investigadores, sem conhecimento prévio sobre as famílias. Os resultados confirmam a validade do instrumento; e indicam que as mães têm conhecimento e acesso ao script de base segura, utilizando-o na produção das suas narrativas.

Palavras-chave: Script de base-segura, modelos internos dinâmicos, vinculação.

\section{The Internal Working Models Analyzed Using the Narrative Assessment of Adult Attachment Representations}

\begin{abstract}
This study analyzed the internal working models of 89 mothers of preschool age children, using the Narrative Assessment of Adult Attachment Representations (Waters \& Rodrigues-Doolabh, 2004). The goals were to test the internal and discriminant validity of the instrument, as well as the stylistic aspects of the narratives produced by the participants. The narratives were collected during individual interviews, transcribed and then coded by 2 to 3 researchers without previous knowledge of these families. Results confirm the validity of the instrument; and show that mothers have knowledge and access to the secure base script to construct their narratives.
\end{abstract}

Keywords: Secure base script, internal working models, attachment.

Endereço para correspondência: Instituto Universitário de Ciências Psicológicas, Sociais e da Vida, Unidade de Investigação Psicologia Cognitiva do Desenvolvimento e Educação, Rua Jardim do Tabaco, 34, Lisboa, Portugal 1149-041. Fone: (+351) 218811 700; Fax: (+351) 218860 954. E-mail: mveriss@ispa.pt e asantos@, ispa.pt Agência de financiamento: Fundação para a Ciência e a Tecnologia (FCT). 


\section{Los Modelos Internos Dinámicos Analizados Mediante las Narrativas de Representación de Apego en Adultos}

\section{Resumen}

Este estudio examinó los modelos internos dinámicos de las madres mediante las Narrativas de Representación de Apego en Adultos (Waters \& Rodrigues-Doolabh, 2004). Los objetivos fueron: probar la validez interna y discriminativa del instrumento, analizando el aspecto estilístico de las narrativas producidas por 89 madres con niños que asisten a la Guardería/ Jardín-de-Infancia. Las narrativas fueron recogidas en sesiones individuales y transcritas y citadas por dos o tres investigadores, sin conocimiento previo acerca de las familias. Los resultados confirman la validez del instrumento; e indican que las madres tienen el conocimiento y acceso al script de base segura, y lo utilizan para la producción de sus narrativas.

Palabras clave: Script de base segura, modelos internos dinámicos, apego.

O conceito de modelo interno dinâmico é definido por Bowlby (1969/1982) como uma componente integrante do sistema de controlo da vinculação; considerando-se que a criança constrói modelos dinâmicos das figuras de vinculação e do self, no contexto das interações diádicas, contínuas e reais com estas figuras. Será com a ajuda destas que a criança interpreta e prevê os comportamentos esperados do outro, e em função das quais planeia as suas respostas atuais e futuras (Bowlby, 1973).

Se durante a infância, os modelos operam primariamente ao nível sensório-motor, com o avançar da idade, e, em particular após os três anos de idade, há uma reorganização para um nível mais simbólico, o que permite à criança refletir e falar sobre os aspectos do comportamento relacionados com as suas figuras vinculativas (Main, Kaplan, \& Cassidy, 1985). Uma vez formados, estes modelos tendem a operar ao nível do inconsciente, esperando-se que permaneçam relativamente estáveis ao longo da vida, embora passíveis de revisão e mudança face a experiências reais relevantes para a vinculação, (Bowlby, 1973, 1988; Waters, Merrick, Treboux, Crowell, \& Albersheim, 2000). Estas representações, que com o desenvolvimento se tornam abstractas e generalizadas, têm impacto ao longo da vida, servindo de modelo implícito para o estabelecimento e funcionamento nas relações afectivas/ íntimas, nomeadamente, onde o fenómeno de base segura está presente, como será o caso das relações românticas e da parentalidade (Bowlby, 1973, 1988; Waters \& Cummings, 2000).

Porém, aquando da introdução da ideia do modelo interno dinâmico, por parte de Bowlby, este não era mais do que uma "metáfora conceptual" (Bretherton, 1985; Hinde, 1988; Thompson, Laible, \& Ontai, 2003), dado pouco se saber acerca da sua organização, funcionamento e desenvolvimento. Apesar dos avanços verificados com a criação da Adult Attachment Interview - AAI (Hesse, 1999; Main et al., 1985) considerada a "medida standard" para a vinculação na idade adulta (na área da psicologia do desenvolvimento), esta é considerada uma avaliação indireta do conteúdo dos modelos internos (state of mind relativo à vinculação). Na perspectiva de Waters e Waters (2006) o passo seguinte (ao nível teórico e empírico) seria a passagem para uma análise mais específica, nomeadamente, ao nível da estrutura cognitiva destes modelos e do seu impacto nos comportamentos, afectos e cognições.

\section{O Script de Base Segura}

No seguimento da sugestão de Bretherton (1985, 1990; Bretherton \& Munholland, 1999) sobre o potencial das teorias das representações de acontecimentos (Nelson, 1986; Schank, 1982; Schank \& Abelson, 1977) para o constructo da vinculação, Waters e Rodrigues-Doolabh (2001) sugerem que a história de interações de base segura é representada/organizada, na memória, sob 
a forma de um script de base segura. Este seria semelhante ao conhecimento de outro tipo de script como por exemplo, uma ida a uma festa de anos (Nelson, 1986). Waters e Rodrigues-Doolabh (2001) desenvolveram uma metodologia que visa, precisamente, avaliar a organização do conhecimento de base segura, utilizando narrativas produzidas por adultos, em resposta a um conjunto de palavras sugestivas, que remetem para interações adulto/criança e adulto/adulto.

Os esquemas de acontecimentos ou scripts são representações gerais de acontecimentos, que partem de uma base configurativa, mas que se vão generalizando, atingindo diferentes níveis de abstração. Contêm informação tipo "esqueleto" acerca de quem, o quê, quando, onde, o porquê e o como dos acontecimentos e preservam aspectos da estrutura causal e espácio-temporal dos acontecimentos reais. Estes são criados no decurso da repetição de experiências, de natureza semelhante, e mobilizados sempre que um determinado acontecimento se aproxima do esquema existente, permitindo ao sujeito prever, com maior ou menor sucesso, o que irá acontecer, para além do contexto imediato, ajudando a preparar e organizar os comportamentos. Ainda que, inicialmente, estes scripts sejam aplicados de um modo voluntário, à medida que o script se torna mais regular, a sua ativação torna-se involuntária - conhecimento implícito (Nelson, 1986; Schank, 1982; Schank \& Abelson, 1977).

Estudos realizados por Waters, Rodrigues e Ridgeway (1998) indicam que as experiências de vinculação, vividas no contexto das primeiras relações, são representadas sob forma de uma estrutura de script causal-temporal em torno dos componentes do "fenómeno de base segura". Waters e Waters (2006) consideram que se o suporte de base segura foi consistente e coerente, o script construído deverá ser completo, bem consolidado e rapidamente acessível em situações relevantes. Se, pelo contrário, foi inconsistente ou ineficaz, o script deverá ser menos bem configurado, e de mais difícil acesso em situações ou interações de base segura. A familiaridade e o acesso a este script, assumem um papel importante na organização do equilíbrio entre os comportamentos de vinculação e os comportamentos de exploração durante os primeiros anos de vida, sendo a base dos modelos internos de vinculação, que emergem posteriormente (Veríssimo, Monteiro, Vaughn, Santos, \& Waters, 2005). $\mathrm{Na}$ idade adulta, o conhecimento de tipo script, relativo a ter e/ou ser uma base segura, contém diversos elementos elaborados na seguinte sequência: (a) interação construtiva entre os membros da díade (adulto/criança ou adulto/adulto); (b) um obstáculo à continuação da interação; (c) um sinal de que é necessária ajuda; (d) detecção do sinal pelo parceiro; (e) oferta de ajuda efetiva; e a ajuda é sentida pelo receptor como reconfortante; (f) resolução e/ou regresso à interação construtiva com o meio físico ou social (Waters \& Rodrigues-Doolabh, 2001). Espera-se, assim, que os sujeitos cujas histórias pessoais se caracterizam por vinculações seguras, tendo por isso tido diversas oportunidades de receber suporte e, provavelmente, de ter servido de base segura a um parceiro (criança e/ou adulto), possuam um script completo e de fácil acesso em situações relevantes para a vinculação.

Estudos realizados em diversas culturas e etnias (Coppola, Vaughn, Cassiba, \& Costantini, 2006; Rodrigues-Doolabh, Zevallos, Turan, \& Green, 2003; Vaughn et al., 2007; Veríssimo et al., 2005; Waters \& Rodrigues-Doohlab, 2001) verificaram que, as representações relativas às relações adulto/criança e adulto/adulto se encontram organizadas num script geral e abstracto para as mães, assim como, para os pais (Monteiro, Veríssimo, Vaughn, Santos, \& Bost, 2008). Vaughn et al. (2006) confirmaram, ainda, a estabilidade das classificações dos scripts_maternos num intervalo de 12/15 meses.

Testando a validade do instrumento, Coppola et al. (2006) e Waters e Rodrigues-Doolabh (2001) verificaram, ainda, que o conhecimento e acesso ao script de base segura se encontra significativamente correlacionado com a escala de coerência da AAI para as mães. Por sua vez Elliott, Tini, Fetten e Sauders (2003) confirmaram esses resultados para adultos do sexo masculino. Em amostras de adolescentes (Dykas, Woodhouse, Cassidy, \& Waters, 2006) as associações encontradas vão no mesmo sentido. O script de base segura materno encontra-se, ainda, significativa- 
mente associado com as classificações dos seus filhos na Situação Estranha (Tini, Corcoran, Rodrigues-Doolabh, \& Waters, 2003) e com a organização dos comportamentos de base segura das crianças em casa (Monteiro et al., 2008; Vaughn et al., 2007; Veríssimo et al., 2005).

Dado o instrumento se basear na produção de narrativas (embora oral), é possível que possam existir associações entre as capacidades verbais dos adultos e a cotação do script de base segura. Neste sentido, Elliott et al. (2003) e Waters e Rodrigues-Doolabh (2001) analisaram as associações entre o QI de mulheres e homens e os valores script de base segura não tendo encontrado correlações significativas. Outros estudos (Monteiro \& Veríssimo, 2010; Waters \& Rodrigues-Doohlab, 2001) verificaram, ainda, que estes valores script não se encontravam significativamente correlacionados com os valores das histórias que remetem para cenários familiares (e.g. uma ida ao parque), mas que do ponto de vista da vinculação são consideradas neutras. As habilitações literárias maternas no estudo de Coppola et al. (2006) e Vaughn et al. (2007) encontravam-se significativamente associadas com o valor do script de base segura contudo, quando controladas, a qualidade do script era preditora da sensibilidade materna e da segurança (Attachment Behaviour Q-Set) das crianças, respectivamente. Estes resultados são indicadores que o instrumento não avalia, pelo menos de um modo significativo, a capacidade dos adultos de produzir narrativas.

\section{Objectivos}

Talvez pelo facto de ser um instrumento relativamente recente, nomeadamente, face à Adult Attachment Interview e, considerando o peso que esta tem na avaliação da vinculação na idade adulta, pouco estudos publicados têm, nos últimos anos, utilizado as Narrativas de Vinculação. Isto apesar dos resultados promissores já descritos. Assim, o presente estudo visou: (a) Contribuir para a análise da validade interna e discriminativa das Narrativas de Representação da Vinculação em Adultos (Waters \& Rodrigues-Doolabh, 2004), dado que apenas um estudo (Monteiro \& Veríssimo, 2010), para além dos trabalhos de Waters e Rodrigues-Doolabh (2001), recorreu à cotação das histórias neutras, cujo conteúdo não se refere a interações de base segura. (b) Na continuidade dos trabalhos de Vaughn et al. (2006) e Monteiro, Fernandes, Veríssimo, Torres e Vaughn (2010) (os únicos estudos publicados que analisam os aspectos estilísticos das narrativas maternas), proceder à análise do número de palavras sugestivas e do número total de palavras utilizadas na produção das histórias (enquanto aspectos organizadores das mesmas) e a sua associação (ou não) com o script de base segura. (c) Analisar a presença e qualidade do script de base segura em narrativas produzidas por mães de crianças a frequentar Creche/Jardim-de-Infância, controlando variáveis sociodemográficas como por e.g. as suas habilitações literárias.

\section{Método}

\section{Participantes}

Participaram do estudo 89 mães, das quais 76 eram casadas, 9 viviam em união de facto e 3 estavam divorciadas. Tinham idades compreendidas entre os 22 e 50 anos $(M=34,96, D P=$ 4,63), variando as suas habilitações literárias entre os 7 e os 23 anos de escolaridade ( $M=$ 15,20, $D P=3,26)$. Do total de mães, 73 delas trabalhavam a tempo inteiro, 3 a tempo parcial, e 13 encontravam-se desempregadas. As crianças tinham idades compreendidas entre os 29 e os 76 meses $(M=38,24, D P=10,92)$, sendo 43 do sexo feminino e 46 do sexo masculino; destas 58 tinham irmãos. As famílias pertenciam a um nível socioeconómico médio e médio/baixo de acordo com os padrões nacionais, tendo sido recrutadas para o projeto através das Creches/Jardins-de-infância que frequentavam, nos Concelhos de Lisboa e Sesimbra. Este estudo faz parte de um projeto longitudinal aprovado pela Comissão de Ética do ISPA - Instituto Universitário.

\section{Instrumentos}

Narrativas de Representação da Vinculação em Adultos (Waters \& Rodrigues-Doolabh, 2004). Quatro grupos de palavras sugestivas foram desenvolvidos com vista a guiar a produção 
de narrativas, relacionadas com cenários importantes para a vinculação e, deste modo, analisar o conhecimento e acesso ao script de base segura, nos adultos, em contextos de rotina e ansiogénicos (Waters \& Rodrigues-Doolabh, 2001). Os dois primeiros, "A manhã do bebé"e "No consultório médico", referem-se às interações adulto/criança, enquanto que, "O acampamento da Joana e do Pedro" e "O acidente da Susana" referem-se às interações adulto/adulto (casal). São, ainda, utilizados dois cenários, baseados em atividades familiares "O passeio no parque" e "Uma tarde nas compras", considerados neutros, uma vez que não são relevantes para o fenómeno de base segura. As palavras sugestivas apresentadas em cada história dão apenas um possível enquadramento para a elaboração da narrativa, pelo que, o mesmo conjunto pode desencadear um diverso número de histórias, igualmente bem elaboradas, em torno do script de base segura (Waters \& Waters, 2006).

As quatro narrativas são cotadas, numa escala de 7 pontos, indicando a extensão em que a narrativa se encontra organizada em torno do script de base segura e a sua riqueza/detalhe relativa à relação entre as personagens da história. Um valor global sintetiza tanto a presença, como a qualidade do script para cada uma das narrativas. Os valores inferiores a 4 indicam a ausência geral do script de base segura. Os valores mais baixos (1-2) são reservados para histórias em que, não só há ausência do script como, também, se verifica a existência de conteúdos bizarros (e.g., a criança que se magoou tranquiliza o pai, que está transtornado com a ferida). Os valores de 4 ou acima na escala de 7 pontos, indicam a presença do script de base segura. Os valores mais elevados são atribuídos quando o script é elaborado, revela conhecimento e sensibilidade face ao estado emocional do outro, reformula o significado do obstáculo/conflito de um modo favorável e/ou integra a interação atual no contexto da relação entre os sujeitos (ver Waters \& Rodrigues-Doolabh, 2004; Waters \& Waters, 2006). A média das 4 histórias, com conteúdo de base segura, indica o valor script de base segura para cada sujeito. As duas histórias neutras são, também, cotadas utilizando a escala de 1 7, com base nos scripts referentes a um passeio no parque e a uma tarde nas compras. De acordo com Waters e Waters (2006), na cotação das narrativas não devem ser tidos em consideração, por exemplo, detalhes relativos à linguagem (e.g., os tempos verbais, ou as repetições) e à veracidade da história (uma vez que todas as histórias são fictícias) devendo evitar-se as inferências acerca dos estados mentais e as interpretações psicodinâmicas.

\section{Procedimento}

Narrativas de Vinculação. As narrativas foram recolhidas numa entrevista individual com a mãe, onde estas responderam a seis conjuntos de palavras sugestivas, cada um com um título da narrativa a contar (Waters \& Rodrigues-Doolabh, 2004) e que foram apresentados, um de cada vez, por um investigador treinado. Para cada história é apresentado um conjunto de palavras organizadas em três colunas. Na primeira, as palavras apresentadas sugerem um possível cenário e os atores iniciais; na segunda alguns conteúdos e atividades; e na última sugerem uma possível conclusão para a história. Foi pedido às mães/pais que lessem cada coluna, de cima para baixo, da esquerda para a direita, de modo a terem uma ideia acerca de uma possível história a contar sendo, no entanto, os pais fossem livres de utilizar as palavras como entendessem. Foram, ainda, informados que as histórias seriam gravadas e que, se assim o entendessem, poderiam parar de contar a história e reiniciá-la.

Apenas quatro das histórias visam desencadear temas relacionados com a vinculação, duas referem-se explicitamente a conteúdos da relação adulto/criança (A manhã do bebé; $\mathrm{O}$ consultório do médico) e duas à temática adulto/ adulto (O acampamento da Joana e do Pedro; $\mathrm{O}$ acidente da Susana). As restantes histórias (O passeio no parque; Uma ida às compras remetem para interações adulto/criança e adulto/ adulto, respectivamente) são consideradas neutras. À medida que os conjuntos de palavras foram sendo apresentados, um por um, o investigador identificava o tema da história e se as 
personagens eram mãe e criança, ou adultos. Estes foram apresentados em seis sequências diferentes, de modo a controlar possíveis efeitos de ordem. As três histórias relativas à interação adulto/criança foram sempre apresentadas como um bloco e as referentes aos adultos como outro bloco, isto é, as mães realizavam todas as três histórias de um bloco, antes de lhes ser apresentada uma história do outro bloco. A maioria das mães realizou a tarefa em 15-20 minutos. As histórias foram, posteriormente, transcritas de forma a serem cotadas.

Dois a três investigadores leram e cotaram cada história, utilizando a escala de 7 pontos definida por Waters e Rodrigues-Doolabh (2004). As histórias foram agrupadas por temas, por exemplo. Todas as histórias de "A Manhã do bebé" foram colocadas num grupo e cotadas na mesma sessão. $\mathrm{O}$ mesmo procedimento foi utilizado para as restantes histórias, cotadas em dias diferentes, de modo a minimizar a possibilidade dos investigadores identificarem as histórias de um único sujeito. Os investigadores receberam treino intensivo na cotação das narrativas por parte de H. Waters. Os valores de correlação entre os investigadores (calculadas como correlações entre classes) variaram entre 0,82 e 0,94 . Os valores Spearman-Brown de fiabilidade para cada história variaram entre 0,79 e 0,84 .

$\mathrm{O}$ valor final para cada narrativa foi obtido através da média dos valores dados pelos investigadores que cotaram as histórias. Efetuou-se a média das cotações das histórias Adulto/criança obtendo-se um valor para este grupo, realizando-se o mesmo para as histórias Adulto/adulto. Um valor global foi obtido através do cálculo da média das 4 histórias com conteúdo de base segura.

Tabela 1

Médias e Desvios Padrão do $\mathrm{N}^{0}$ de Palavras e do $\mathbf{N}^{0}$ Total de Palavras para as Histórias Adulto/Criança, Adulto/Adulto e Neutras

\begin{tabular}{lccc}
\hline Aspectos estilísticos & Adulto/Criança & Adulto/ Adulto & Neutras \\
\hline $\mathrm{N}^{\mathrm{o}}$ palavras sugestivas & $18,1(9,92)$ & $15,49(6,78)$ & $17,45(9,1)$ \\
$\mathrm{N}^{\mathrm{o}}$ total palavras & $188,97(105,92)$ & $202,39(131,67)$ & $197,55(126,34)$ \\
\hline
\end{tabular}

Foram efetuadas análises de correlação de Pearson entre as diferentes variáveis, assim como, análises de $t$ de student, de modo a responder aos objectivos do estudo.

\section{Resultados}

Analisaram-se as relações intra histórias adulto/criança e adulto/adulto e entre os dois compósitos. Obtiveram-se correlações positivas e significativas nas histórias adulto/criança, $r(89)=0,48, p<0,001$; nas histórias adulto/adulto, $r(89)=0,47, p<0,001$; e entre os compósitos adulto/criança e adulto/adulto, $r(89)=0,63$, $p<0,001$. O valor do Alfa de Cronbach para as histórias com script de base segura é de 0,76 , o que traduz níveis aceitáveis de consistência interna do instrumento, sendo comparável aos obtidos noutros estudos (e.g., Elliott et al., 2003; Monteiro et al., 2008; Rodrigues-Doohlab et al., 2003; Veríssimo, Monteiro, \& Santos, 2006).

\section{Validade Discriminativa - Histórias Neutras}

Correlacionaram-se os valores script do compósito das histórias adulto/criança e adulto/ adulto com o compósito das histórias neutras tendo-se, apenas, obtido uma associação significativa com as histórias adulto/adulto, $r(89)=$ $0,30, p<0,05$.

\section{Aspectos Estilísticos das Narrativas Maternas}

Analisou-se o número de palavras sugestivas e o número total de palavras utilizados pelas mães na elaboração das suas narrativas. As Médias e os Desvios Padrão são apresentados na Tabela 1. 
Análises $t$-student indicam a existência de diferenças significativas apenas entre o número de palavras sugestivas utilizadas pelas mães nas narrativas adulto/criança e adulto/adulto, $t(86)=$ 4,$38 ; \mathrm{p}<0,001$.

\section{Script de Base Segura Materno}

As narrativas maternas foram analisadas, quanto à presença e qualidade do script de base segura, nas histórias adulto/criança $(M=3,97$; $D P=1,14)$, adulto/adulto $(M=3,66 ; D P=1,04)$ e no Script total $(M=3,82 ; D P=0,96)$. Os valores obtidos indicam que, face a um conjunto de palavras sugestivas que remetem para conteúdos específicos da vinculação, as mães acedem e utilizam o script de base segura na produção das suas narrativas.

\section{Análise das Variáveis Sociodemográfi- cas}

Analisaram-se as relações entre as variáveis sociodemográficas: idade e habilitações literárias da mãe; idade da criança e o valor script de base segura (total) materno, não tendo sida encontrada qualquer correlação significativa. Análises $t$-student para amostras independentes indicam que não existem diferenças significativas nos valores script de base segura das mães em função do sexo da criança, ou das crianças terem ou não irmãos.

\section{Análise das Relações entre os Aspec- tos Estilísticos e o Script de Base Segu- ra Materno}

Existem correlações significativas entre o número total de palavras sugestivas nas histórias adulto/criança e o valor script de base segura $(r=0,31, p<0,01)$, e o número total de palavras utilizadas pelas mães nas histórias adulto/ criança, $\quad r(87)=0,52, \quad p<0,001, \quad$ adulto/adulto, $r(87)=0,35, p<0,001$ e o valor script de base segura.

\section{Discussão e Conclusão}

A introdução do conceito de modelo interno dinâmico (Bowlby, 1973), na teoria da vincula- ção, tornou possível a análise do fenómeno de base segura ao longo da vida, permitindo compreender as mudanças que ocorrem nas suas expressões, assim como, o seu impacto no desenvolvimento e adaptação psicossocial do indivíduo (Crowell, Fraley, \& Shaver, 1999). Dado o impacto e peso que AAI teve, e continua a ter, no estudo da vinculação na idade adulta, pode haver a suspeição de que as Narrativas de Vinculação sejam apenas mais um instrumento da mesma família, que procura evitar um processo moroso e trabalhoso de treino, realização, transcrição e cotação dos protocolos da AAI (Bakermans-Kranenburg, 2006).

Waters e Rodrigues-Doohlab (2001, 2004) criaram um instrumento que procura avaliar as diferenças individuais no conhecimento e acesso ao script de base segura, mantendo desta forma a ligação ao conceito central da teoria da vinculação: o fenómeno de base segura (Waters \& Cummings, 2000). Para Waters (2003), tal não significa que as estruturas do tipo script sejam o único modo pelo qual as experiências iniciais são representadas. Significa apenas que estas são adequadas para representar as interações de vinculação, têm características de funcionamento conhecidas e são empiricamente acessíveis.

Visando contribuir para a compreensão e validação deste instrumento, realizaram-se análises ao nível da validade interna, indicando que as correlações intra e inter histórias Adulto/criança e Adulto/adulto são comparáveis às apresentadas por Rodrigues-Doohlab et al. (2003) e Vaughn et al. (2007), assim como, por Monteiro e Veríssimo (2010) e Veríssimo et al. (2005) em amostras portugueses. Os valores de fiabilidade do compósito de base segura são aceitáveis (e.g., Monteiro \& Veríssimo, 2010; Rodrigues-Doohlab et al., 2003; Vaughn et al., 2007; Veríssimo et al., 2005).

Relativamente à validade discriminativa, no geral, os resultados vão no sentido dos estudos iniciais de Monteiro e Veríssimo (2010) e de Waters e Rodrigues-Doohlab (2001), indicando que este instrumento não avalia, pelo menos de um modo significativo, a capacidade dos adultos produzirem narrativas. No entanto, o valor script de base segura para as histórias adulto/adulto, 
contrariamente aos estudos anteriores, encontra-se significativamente correlacionado com o valor das histórias neutras. Refira-se, ainda, que não existem associações significativas entre as habilitações literárias de mães e os valores script de base segura. $\mathrm{O}$ facto de as histórias serem produções orais ajuda a minimizar as possíveis diferenças individuais em termos de estilos e competência de produção escrita (Waters \& Waters, 2006). Contudo, na perspectiva de Bakermans-Kranenburg (2006), enquanto pedras basilares dos modelos internos dinâmicos de vinculação, os scripts, como estruturas cognitivas que são e pelo facto de serem avaliados através de narrativas, a sua eventual associação com a competência verbal dos sujeitos não deverá ser encarada como algo inesperado.

As análises realizadas indicam que as mães têm conhecimento e acesso ao script de base segura. Face a diferentes conjuntos de palavras sugestivas, as crenças e expectativas das mães, relacionadas com o script, são ativadas e guiam as suas produções narrativas. Alguns adultos têm expectativas diferentes, ou menos consistentes acerca destas interações, não compreendendo a implicação do conhecimento de base segura, mesmo ao longo dos diversos conjuntos de palavras sugestivas (Waters \& Waters, 2006). A ideia implícita ao uso das narrativas é que os processos mentais variam, tal como os processos comportamentais, em função dos diferentes modelos internos, o que se reflete na linguagem (Crowell et al., 1999; Main et al., 1985). Tal tem implicações na distribuição das classificações dos sujeitos ao longo da escala de 7 pontos definida por Waters e Rodrigues-Doolabh (2004).

$\mathrm{Na}$ análise dos aspectos estilísticos das narrativas, obtiveram-se correlações significativas entre o número total de palavras utilizadas pelas mães nas diferentes narrativas e o script de base segura, o que não seria, necessariamente, espectável, uma vez que os valores script não são função da dimensão da história contada. Estes dados não confirmam os resultados obtidos nos estudos de Monteiro e Veríssimo (2010) indo, no entanto, ao encontro dos resultados de Vaughn et al. (2006). Estes resultados refletem, pelo menos em parte, a necessidade da elaboração e introdução de detalhes nas narrativas. Refirase que, histórias com menos de 75 palavras recebem, normalmente, cotações de " 3 " ou menos, uma vez que não conseguem estabelecer a relação entre as personagens e fornecer algum detalhe acerca do significado do acontecimento da história. Histórias que recebem o valor mais elevado "7" fornecem um detalhe considerável acerca da relação entre os elementos da díade, do estado emocional e dos comportamentos dos mesmos, no contexto da situação sugerida, pelo que têm que ser relativamente longas (> 250 palavras; Vaughn et al., 2006; Vaughn et al., 2007). Tal não significa que histórias longas tenham, necessariamente, conteúdo de base segura, ou que, histórias relativamente curtas não possam conter, pelo menos, alguns elementos de base segura. Apenas para as histórias adulto/ criança se encontram associações significativas com o número de palavras sugestivas utilizadas. No estudo de Monteiro e Veríssimo (2010) não existem associações com as narrativas maternas, apenas paternas. Vaughn et al. (2006) interpretam a mesma associação por eles encontrada, numa amostra de mães americanas, referindo a possibilidade dos sujeitos que utilizam o script de base segura, na construção das histórias, serem capazes de o reconhecer nas palavras sugestivas, incorporando-as nas suas narrativas. Porém, isso não se verifica nas narrativas adulto/ adulto, pelo que estudos adicionais são aconselháveis.

\section{Considerações Finais}

Os resultados obtidos indicam que as Narrativas de Representação da Vinculação em Adultos são um instrumento válido e útil na avaliação das representações de tipo script de base segura em adultos, nomeadamente, portugueses. Estas permitem, não só uma análise mais específica, nomeadamente, ao nível da estrutura cognitiva dos modelos internos dinâmicos, como do ponto de vista do tempo de recolha e cotação são uma medida, claramente, mais económica, comparativamente com a Adult Attachment Interview.

Trabalhos futuros deverão alargar as suas análises ao estudo de adultos do sexo masculino 
(e.g. Monteiro et al., 2010), dada a importância cada vez maior do estudo da figura paterna na área da vinculação. Considera-se, ainda, importante aplicar este instrumento a amostras com maior variabilidade ao nível dos anos de escolaridade e do estatuto socioeconómico; controlando-se o QI verbal ou Índice de Competência Verbal dos sujeitos.

\section{Referências}

Bakermans-Kranenburg, M. J. (2006). Script-like attachment representations: Steps towards a secure base for further research. Attachment and Human Development, 8, 275-281. doi:10.1080/14616730600910037

Bowlby, J. (1973). Attachment and loss: Vol. 2. Separation, anxiety, and anger. New York: Basic Books.

Bowlby, J. (1982). Attachment and loss: Vol. 1. Attachment ( $2^{\text {nd }}$ Rev. Ed.). New York: Basic Books (Original work published 1969)

Bowlby, J. (1988). A secure base. Parent-child attachment and healthy human development. New York: Basic Books.

Bretherton, I. (1985). Attachment theory: Retrospect and prospect. Monographs of the Society for Research in Child Development, 50(1/2), 3-39. doi: $10.2307 / 3333824$

Bretherton, I. (1990). Communication patterns, internal working models and the Intergenerational transmission of attachment relationships. Infant Mental Health Journal, 11-3, 237-252. doi:10.1 002/10970355(199023)11:3\%3C237::AIDIMH J2280110306\%3E3.0.CO;2X

Bretherton, I., \& Munholland, K. A. (1999). Internal working models in attachment relationships: A construct revisited. In J. Cassidy \& P. R. Shaver (Eds.), Handbook of attachment: Theory, research, and clinical applications (pp. 89-111). New York: Guilford.

Coppola, G., Vaughn, B. E., Cassibba, R., \& Costantini, A. (2006). The attachment script representation procedure in an Italian sample: Associations with Adult Attachment Interview scales and maternal sensitivity. Attachment and Human Development, 8, 209-219. doi:10.1080/14616730600856065
Crowell, J. A., Fraley, R. C., \& Shaver, P. R. (1999). Measurement of individual differences in adolescent and adult attachment. In J. Cassidy \& P. R. Shaver (Eds.), Handbook of attachment: Theory, research, and clinical applications (pp. 434-465). New York: Guilford.

Dykas, M. J., Woodhouse, S. S., Cassidy, J., \& Waters, H. S. (2006). Narrative assessment of attachment representations: Links between secure base scripts and adolescent attachment. Attachment and Human Development, 8, 221-240. doi:10.1080/14616730600856099

Elliott, M., Tini, M., Fetten, E., \& Saunders, A. (2003, March). Attachment scripts in adult man and adolescent males. In H. Waters \& E. Waters (Chairs), Script-like representations of secure base experience: Evidence of cross-age, cross-cultural, and behavioral links. Poster symposium presented at the Biennial Meetings of the Society for Research in Child Development, Tampa, FL, United States

Hesse, E. (1999). The adult attachment interview: Historical and current perspectives. In J. Cassidy \& P. R. Shaver (Eds.), Handbook of attachment: Theory, research, and clinical implications (pp. 395-433). New York: Guilford.

Hinde, R. A. (1988). Continuities and discontinuities. In M. Rutter (Ed.), Studies of psychosocial risk: The power of longitudinal data (pp. 367-384). Cambridge, UK: Cambridge University Press.

Main, M., Kaplan, N., \& Cassidy, J. (1985). Security in infancy, childhood, and adulthood. A move to the level of representation. Monographs of the Society for Research in Child Development, 50(1/2), 66-104. doi:10.2307/3333827

Monteiro, L., Fernandes, M., Veríssimo, M., Torres, N., \& Vaughn, B. E. (2010). Perspectiva do pai acerca do seu envolvimento em famílias bi-parentais: Associações com o que é desejado pela mãe e com as características da criança. Interamerican Journal of Psychology, 44, 120-130.

Monteiro, L., \& Veríssimo, M. (2010). Análise do fenómeno de base segura em contexto familiar: $A$ especificidade das relações criança/mãe $e$ criança/pai. Textos Universitários de Ciências Sociais e Humanas. Lisboa, Portugal: Fundação Calouste Gulbenkian.

Monteiro, L., Veríssimo, M., Vaughn, B. E., Santos, A., \& Bost, K. K. (2008). Secure base representations for both fathers and mothers predict 
children's secure base behavior in a sample of Portuguese families. Attachment and Human Development, 10, 189-206.

Nelson, K. (1986). Event knowledge: Structure and function in development. Hillsdale, NJ: Erlbaum.

Rodrigues-Doolabh, L., Zevallos, A., Turan, B., \& Green, K. (2003, March). Attachment scripts across cultures: Further evidence for a universal secure base script. In H. Waters \& E. Waters (Chairs), Script-like representations of secure base experience: Evidence of cross-age, cross-cultural, and behavioral links. Poster symposium presented at the Biennial Meetings of the Society for Research in Child Development, Tampa, FL, United States.

Schank, R. C. (1982). Dynamic memory: A theory of reminding and learning in computers and people. New York: Cambridge University Press.

Schank, R. C., \& Abelson, R. P. (1977). Scripts, plans, goals and understanding. Hillsdale, NJ: Erlbaum.

Thompson, R. A., Laible, D. J., \& Ontai, L. L. (2003). Early understanding of emotion, morality, and the self: Developing a working model. In R. V. Kail (Ed.), Advances in child development and behavior (Vol. 31, pp. 137-171). San Diego, CA: Academic.

Tini, M., Corcoran, D., Rodrigues-Doolabh, L., \& Waters, E. (2003, March). Maternal attachment scripts and infant secure base behavior. In $\mathrm{H}$. Waters \& E. Waters (Chairs), Script-like representations of secure base experience: Evidence of cross-age, cross-cultural, and behavioral links. Poster symposium presented at the Biennial Meetings of the Society for Research in Child Development, Tampa, FL, United States.

Vaughn, B. E., Copolla, G., Veríssimo, M., Monteiro, L., Santos, A. J., Posada, G., ...Korth, B. (2007). Coordination between the organization of mothers' secure base knowledge and their children's secure base behavior at home. International Journal of Behavioral Development, $31,65-76$

Vaughn, B. E., Veríssimo, M., Coppola, G., Bost, K. K., Shin, N., McBride, B., ...Korth, B. (2006). Maternal attachment script representations: Longitudinal stability and associations with stylistic features of maternal narratives. Attachment and Human Development, 8, 199-208. doi:10.1080/14616730600856024
Veríssimo, M., Monteiro, L., \& Santos, A. J. (2006). Para além da mãe: Vinculação na tríade mãe-pai-criança. In J. C. Coelho Rosa \& S. Sousa (Eds.), Caderno do bebé (pp. 73-85). Lisboa, Portugal: Fim de Século.

Veríssimo, M., Monteiro, L, Vaughn, B. E., Santos, A. J., \& Waters, H. (2005). Coordenação entre o modelo interno dinâmico da mãe e o comportamento de base segura dos seus filhos. Análise Psicológica, 23, 7-17.

Waters, E. (2003, March). Script-like representations of secure base experience: Evidence of cross-age, cross-cultural, and behavioral links. Poster symposium presented at the Biennial Meetings of the Society for Research in Child Development, Tampa, FL, United States.

Waters, E., \& Cummings, E. M. (2000). A secure base from which to explore close relationships. Child Development, 71, 164-172. doi:10.1111/14678624.00130

Waters, E., Merrick, S., Treboux, D., Crowell, J., \&Albersheim, L. (2000). Attachment stability in infancy and in early adulthood: A 20-years longitudinal study. Child Development, 71, 684689.

Waters, H. S., \& Rodrigues-Doolabh, L. (2001, April). Are attachment scripts the building blocks of attachment representations? Narrative assessment of representations and the AAI. Poster symposium presented at the Biennial Meetings of the Society for Research in Child Development, Minneapolis, MN, United States.

Waters, H. S., \& Rodrigues-Doolabh, L. M. (2004). Manual for decoding secure base narratives. Unpublished manuscript, State University of New York at Stony Brook, NY, United States.

Waters, H. S., Rodrigues, L. M., \& Ridgeway, D. (1998). Cognitive underpinnings of narrative attachment assessment. Journal of Experimental Child Psychology, 71, 211-234. doi:10.1006/ jecp.1998.2473

Waters, H. S., \& Waters, E. (2006). The attachment working model concept: Among other things, we built script-like representations of secure base experiences. Attachment and Human Development, 8, 185-197. 